Article

\title{
Assessment of the in Vitro Antithrombotic Properties of Sardine (Sardina pilchardus) Fillet Lipids and Cod Liver Oil
}

\section{Gregory Morphis ${ }^{1, \dagger}$, Aggeliki Kyriazopoulou ${ }^{1, \dagger}$, Constantina Nasopoulou ${ }^{2, \dagger}$, Eleni Sioriki ${ }^{1, \dagger}$, Constantinos A. Demopoulos ${ }^{3}$ and Ioannis Zabetakis ${ }^{1, *}$}

1 Laboratory of Food Chemistry, Faculty of Chemistry, School of Sciences, National and Kapodistrian University of Athens, Athens 15771, Greece;

E-Mails: gregmorphis@chem.uoa.gr (G.M.); aggelikikiriazopoulou@hotmail.com (A.K.); esioriki@gmail.gr (E.S.)

2 Department of Food Science and Nutrition, School of the Environment, University of the Aegean, Myrina Lemnos 81400, Greece; E-Mail: knasopoulou@aegean.gr

3 Laboratory of Biochemistry, Faculty of Chemistry, School of Sciences, National and Kapodistrian University of Athens, Athens 15771, Greece; E-Mail: demopoulos@chem.uoa.gr

$\dagger$ These authors contributed equally to this work.

* Author to whom correspondence should be addressed; E-Mail: izabet@chem.uoa.gr; Tel.: +30-210-7274-663; Fax: +30-210-7274-476.

Academic Editor: Jana Pickova

Received: 7 July 2015 / Accepted: 17 September 2015 / Published: 28 September 2015

Abstract: The aim of the current study was to compare the biological activities of total polar lipids (TPL) and thin-layer chromatography (TLC) polar lipid fractions of sardine fillet and cod liver oil against atherogenesis. TPL and TLC polar lipid fractions obtained from these two sources were assessed for their ability to inhibit the platelet-activating-factor (PAF)-induced platelet aggregation (PAF-antagonists) or to induce platelet aggregation (PAF-agonists), since PAF plays a crucial role in the initiation and development of atherosclerosis. This study focused on the polar lipids since previous studies have underlined that the antithrombotic properties of foodstuffs are mainly attributed to polar lipid micro-constituents. TPL of sardine fillet induced platelet aggregation, while TPL of cod liver had a bimodal effect on platelets. TLC polar lipid fractions of both samples exhibited in vitro aggregatory and inhibitory activity towards platelets. However, TLC sardine polar lipid fractions showed stronger in vitro antithrombotic activities than the cod liver oil ones. These data constitute evidence of the putative contribution of fish polar lipids against 
cardiovascular diseases, underling firstly the beneficial effect of fish and fish lipids as functional foodstuffs against atherogenesis and secondly the more important role of sardine polar lipids as opposed to cod liver oil.

Keywords: sardine (Sardina pilchardus); cod liver oil; antithrombotic properties; cardiovascular diseases

\section{Introduction}

The main beneficial health effect of fish and fish oil consumption has been attributed to their protective activity against cardiovascular diseases (CVD), which has been demonstrated by epidemiological studies [1]. Fish oil is a good source of two important $\omega-3$ polyunsaturated fatty acids (PUFA):

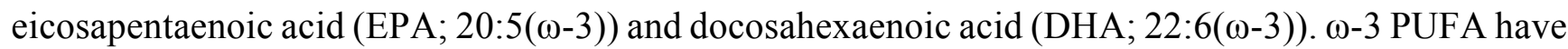
been found to exert potential protective activity against thrombosis and cardiovascular diseases [2]. Additionally, it has been suggested that other substances, apart from $\omega-3$ PUFAs, could be responsible for the antithrombotic properties of marine fish $[3,4]$. Scientific data reported the presence of lipid micro-constituents in different fish species that have been found to exert in vitro anti-thrombotic properties [5-8] and in vivo anti-atherogenic activity [9].

One of the most common global causes of sudden deaths the last decades has been CVD [10]. PAF (1-O-alkyl-2-acetyl-sn-glycero-3-phosphocholine) is an endogenous synthesized phospholipid compound [11], which has been characterized as a potent inflammatory mediator with a crucial role to the mechanism of atherogenesis [12].

Recent studies that have been conducted on polar lipid fractions of sea bass (Dicentrarchus labrax) [13] and gilthead sea bream (Sparus aurata) [14] fed with olive pomace enriched fish feed exhibited potent antithrombotic properties. Therefore, fish and fish oil are considered to be functional foodstuffs possessing protective properties against CVD.

Sardine (Sardina pilchardus) is an important Mediterranean commercial fish species. It is a fatty fish that stores its fats as triacylglycerols in the flesh. It is also a good source of fat-soluble vitamins and high quality proteins, while sardine fillet lipids have important nutritional characteristics because of their high level of $\omega$-3 PUFA [15].

Several formulations of $\omega-3$ PUFA on the market are manufactured from sardine fish oil through a complex process of purification, during which environmental pollutants and cholesterol are removed, bleaching and concentration (molecular distillation and urea complexation) of high grade sardine oil. These concentrated products contain a total concentration of $90 \% \omega-3$ ethyl esters.

Previous studies in our laboratory showed that those formulations of $\omega$-3 PUFA include some microconstituents that can induce washed rabbit platelet aggregation or inhibit the PAF-induced platelet aggregation. Given this background work, in this paper, we have chosen to study further sardine (Sardina pilchardus) lipids and more specifically the total polar lipids (TPL) of sardine fillet.

Cod (Gadus morhua), a coldwater marine fish, is an important source of EPA and DHA, fat-soluble vitamins and high quality protein. Cod is a lean fish that stores its reserve fats as 
triacylglycerols in the liver [16,17]. Its liver contains $50 \%-60 \%$ fat and accounts for $8 \%-12 \%$ of the total weight of the fish [18].

Cod liver oil is a well-known "nutraceutical", which contains a wide range of substances, including triacylglycerols, mono- and di-acylglycerols, free fatty acids, $\omega-3$ PUFA [19], and it is a major natural source of vitamins A and D [20]. It is widely used as a dietary supplement. Scientific data have demonstrated that compounds with strong PAF-like and anti-PAF activity have been found in cod (Gadus morhua) [6]. All polar lipid fractions of cod have been found to inhibit, in a dose-dependent manner, PAF-induced aggregation or induce platelet aggregation [6]. Bearing in mind this necessity for further studies, we have chosen to study cod liver lipids and more specifically its polar lipid fractions.

Therefore, the aim of our study was to evaluate and compare the in vitro biological activities of (a) cod liver oil produced by MERCK as a dietary supplement and (b) sardine fillet lipids extracted in our laboratory, against platelet aggregation and hence atherogenesis.

\section{Results and Discussion}

\subsection{Total Lipids (TL), Total Polar Lipids (TPL) and Total Neutral Lipids (TNL) of Sardine} (Sardina pilchardus) Fillet Lipids and Cod Liver Oil

The procedure followed for the extraction of sardine fillet TL, along with the separation of sardine fillet TL and cod liver oil into TPL and TNL is shown in Figure 1.

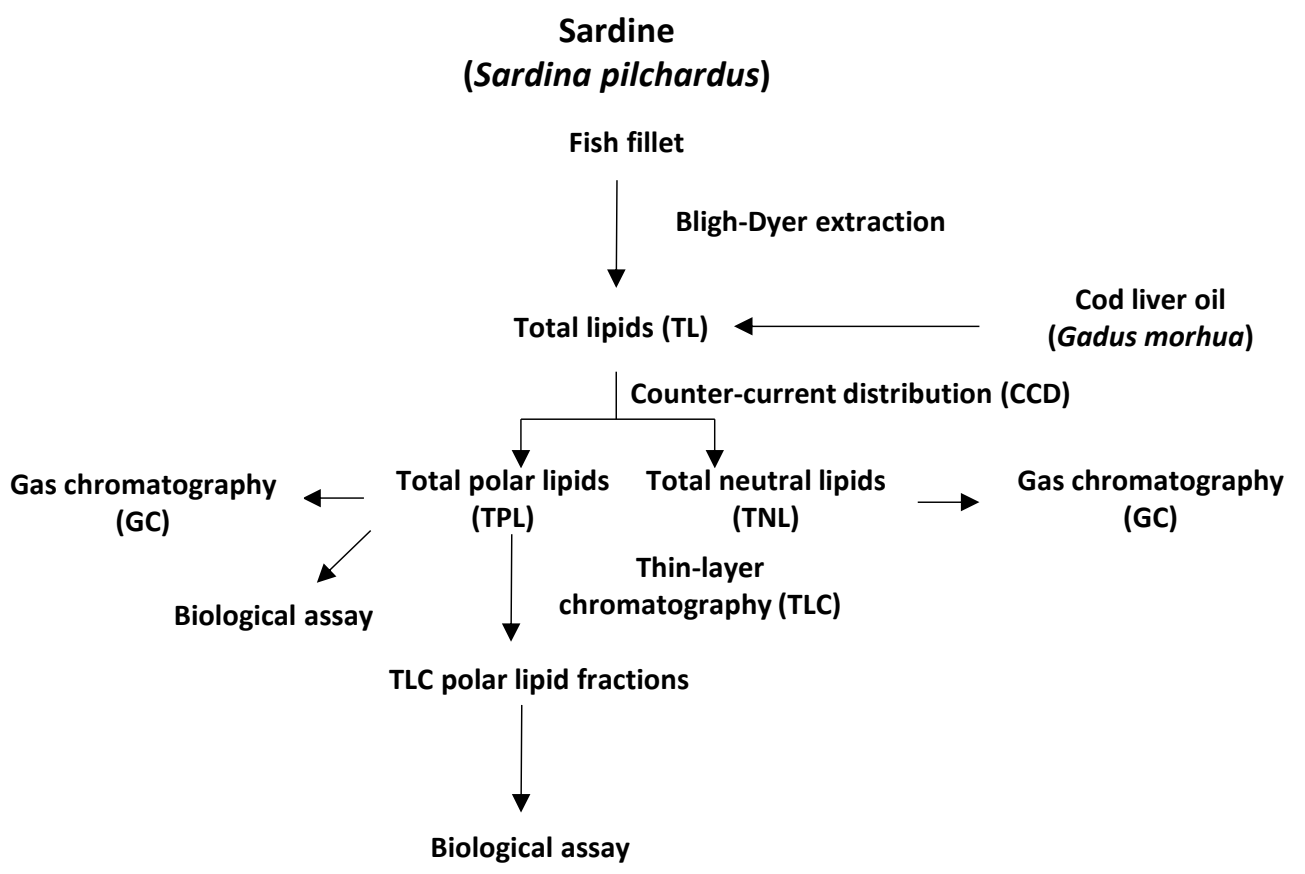

Figure 1. Schematic diagram of the experimental procedure that was followed.

The extraction of sardine fillet TL was carried out by the method of Bligh and Dyer [21], which had been used for the extraction of other fish lipids [6-8]. The obtained TL of sardine fillet $(1.03 \mathrm{~g} \pm 0.02)$ was expressed as $\mathrm{g} / 100 \mathrm{~g}$ of fish tissue $(0.31 \mathrm{~g} \pm 0.006)$.

An amount (1/10) of TL of sardine fillet was stored at $-20{ }^{\circ} \mathrm{C}$. The rest of TL of sardine fillet along with a quantity of $30 \mathrm{~g}$ of cod liver oil, which had been supplied by Merck (Darmstadt, Germany), were 
separated into TPL and TNL by counter current distribution chromatography (CCD) [22]. This method allows excellent recovery of polar lipids from neutral sources. CCD distribution method allows the retrieval of polar lipid fraction containing glyco- and phospholipids. The obtained amounts of TPL and TNL of both samples; sardine fillet and cod liver, were expressed as $\mathrm{g} / 100 \mathrm{~g}$ of fish lipids or oil and the results are shown in Table 1.

Table 1. Total polar lipids (TPL) and total neutral lipids (TNL) content of sardine fillet (g/100 $\mathrm{g}$ fish lipid) and TPL and TNL content of cod liver ( $\mathrm{g} / 100 \mathrm{~g}$ fish oil) (means $\pm \mathrm{SD}$; $n=6)$.

\begin{tabular}{ccc}
\hline Sample & $\begin{array}{c}\text { TPL } \\
\text { (g/100 g Fish Lipids/Oil) }\end{array}$ & $\begin{array}{c}\text { TNL } \\
(\mathbf{g} / \mathbf{1 0 0} \mathbf{g} \text { Fish Lipids/Oil) }\end{array}$ \\
\hline Sardine fillet lipids & $57.7 \pm 1.27^{\mathrm{a}, *}$ & $31.2 \pm 0.65^{\mathrm{a}, * *}$ \\
Cod liver oil & $1.00 \pm 0.03^{\mathrm{b}, *}$ & $93.6 \pm 2.81^{\mathrm{b}, * *}$ \\
\hline
\end{tabular}

Values are means of three individual measurements. Results are expressed as mean \pm SD. $a, b$ indicate significantly different values between the two different fish samples (sardine fillet lipids $v s$. cod liver oil; $p<0.05$ ) according to the Mann-Whitney $U$-test. *, ** indicate significantly different values within the same fish sample between lipid fractions (TPL $v s$. TNL; $p<0.05$ ) according to the Wilcoxon test.

TPL content of sardine fillet was found to be statistically higher than that of cod liver. Furthermore, TNL content of sardine fillet was found to be significantly lower than that of cod liver.

Regarding the TPL and TNL content of cod liver, the amount of TNL was found to be statistically higher than the amount of TPL, which is in accordance with the literature, where neutral lipids have been found to be the dominant lipid class in cod liver [16].

TPL content of sardine fillet was found to be significantly higher compared to the one of TNL, probably due to the fact that only the fish consumable tissue and not the fish head, viscera and bones, which contain higher amounts of TNL [23,24], had been extracted. In addition, the lipid composition of cod [25] and sardine $[15,26]$ has been found to vary due to several factors such as age, sexual maturation, feeding and fasting state, activity level, size, genetics, nutrient composition and energy content of the diets, seasonal change and area of capture.

\subsection{Fatty Acid Profile of TPL and TNL of Sardine Fillet and Cod Liver}

The fatty acid composition of TPL and TNL of sardine fillet $(\mathrm{mg} / \mathrm{kg}$ of fish lipids) and cod liver (mg/kg of fish oil) are summarized in Tables 2 and 3, respectively.

The dominant SFA of both sardine fillet and cod liver TPL was found to be 16:0, while the dominant MUFA was found to be 18:1 cis (Table 2). Such results are in accordance with the literature, where the dominant SFA and MUFA of sardine fillet TPL [26] and cod liver [16], were found to be 16:0 and 18:1 cis, respectively.

Regarding the polyunsaturated fatty acids (PUFA) of sardine fillet TPL, the dominant PUFA was found to be 22:6 ( $\omega-3)$, which was in agreement to literature [26], while the dominant PUFA of cod liver TPL was found to be 20:5 ( $\omega-3)$ (Table 2). 
Distribution of fatty acids in TPL of sardine fillet and cod liver were found to be PUFA $>$ SFA $>$ MUFA and PUFA $>$ MUFA $>$ SFA, respectively (Table 2), findings which were in accordance with the literature, pointing out the same distribution of lipid classes of sardine fillet [26] and cod liver [16].

Additionally, fatty acid levels of TPL of sardine fillet were found to be significantly elevated compared to the ones of cod liver (Table 2).

Table 2. Fatty acid composition of TPL (means $\pm \mathrm{SD} ; n=6$ ) of sardine fillet and cod liver, expressed in $\mathrm{mg} / \mathrm{kg}$ of fish lipid/oil.

\begin{tabular}{ccc}
\hline Fatty Acid & $\begin{array}{c}\text { Sardine Fillet Lipid } \\
\text { (mg/kg of Fish Lipid) }\end{array}$ & $\begin{array}{c}\text { Cod Liver Oil } \\
\text { (mg/kg of Fish Oil) }\end{array}$ \\
\hline $14: 0$ & n.d. & $33.3 \pm 0.99$ \\
$16: 0$ & $6011 \pm 1421^{\text {a }}$ & $69.0 \pm 0.80^{\mathrm{b}}$ \\
$16: 1(\omega-7)$ & n.d. & $53.3 \pm 0.87$ \\
$18: 0$ & $927 \pm 4.72^{\mathrm{a}}$ & $12.8 \pm 0.07^{\mathrm{b}}$ \\
$18: 1$ cis $(\omega-9)$ & $778 \pm 223^{\mathrm{a}}$ & $69.9 \pm 0.25^{\mathrm{b}}$ \\
$18: 1$ trans $(\omega-9)$ & $60.3 \pm 84.7^{\mathrm{a}}$ & $8.48 \pm 1.37^{\mathrm{b}}$ \\
$18: 2(\omega-6)$ & n.d. & $11.5 \pm 0.61$ \\
$18: 3(\omega-3)$ & n.d. & $7.16 \pm 0.72$ \\
$20: 4(\omega-6)$ & $352 \pm 6.30^{\mathrm{a}}$ & $1.46 \pm 0.05^{\mathrm{b}}$ \\
$20: 5(\omega-3)$ & $1527 \pm 19.9^{\mathrm{a}}$ & $71.3 \pm 0.44^{\mathrm{b}}$ \\
$22: 5(\omega-3)$ & $75.8 \pm 0.74^{\mathrm{a}}$ & $5.03 \pm 0.23^{\mathrm{b}}$ \\
$22: 6(\omega-3)$ & $14,077 \pm 120^{\mathrm{a}}$ & $64.7 \pm 1.94^{\mathrm{b}}$ \\
Total $\omega-3$ & $15,680 \pm 141^{\mathrm{a}}$ & $148 \pm 3.32^{\mathrm{b}}$ \\
Total $\omega-6$ & $352 \pm 6.30^{\mathrm{a}}$ & $13.0 \pm 0.66^{\mathrm{b}}$ \\
Total $\omega-7$ & n.d. & $53.3 \pm 0.87$ \\
Total $\omega-9$ & $838 \pm 308^{\mathrm{a}}$ & $78.4 \pm 1.62^{\mathrm{b}}$ \\
Total SFA & $6938 \pm 6.14^{\mathrm{a}}$ & $115 \pm 1.86^{\mathrm{b}}$ \\
Total MUFA & $838 \pm 308^{\mathrm{a}}$ & $132 \pm 2.49^{\mathrm{b}}$ \\
$\omega-6 / \omega-3$ & $0.022^{\mathrm{b}}$ & 0.087 \\
\hline
\end{tabular}

n.d.: not detectable; SFA: saturated fatty acids; MUFA: monounsaturated fatty acids; $\mathrm{a}, \mathrm{b}$ indicate significantly different values between the two fish samples (sardine fillet lipids $v s$. cod liver oil; $p<0.05$ ) according to the Mann-Whitney $U$-test.

Regarding the fatty acid content of both sardine fillet and cod liver TNL, the dominant SFA, MUFA were found to be 16:0, 18:1 cis, respectively, which was in agreement with the findings of other researchers [16,26], while the dominant PUFA of both sardine fillet and cod liver TNL was found to be 22:6 ( $\omega-3)$, (Table 3).

Distribution of fatty acids in TNL of sardine fillet and cod liver were found to be PUFA $>$ SFA $>$ MUFA and MUFA $>$ PUFA $>$ SFA, respectively (Table 3). 
Table 3. Fatty acid composition of TNL (means $\pm \mathrm{SD} ; n=6$ ) of sardine fillet and cod liver, expressed in $\mathrm{mg} / \mathrm{kg}$ of fish lipid/oil.

\begin{tabular}{ccc}
\hline Fatty Acid & $\begin{array}{c}\text { Sardine Fillet Lipid } \\
\text { (mg/kg of Fish Lipid) }\end{array}$ & $\begin{array}{c}\text { Cod Liver Oil } \\
(\mathbf{m g} / \mathbf{k g} \text { of Fish Oil })\end{array}$ \\
\hline $14: 0$ & $16.8 \pm 5.43^{\mathrm{a}}$ & $123 \pm 24.7^{\mathrm{b}}$ \\
$16: 0$ & $843 \pm 12.3^{\mathrm{a}}$ & $635 \pm 16.5^{\mathrm{b}}$ \\
$16: 1(\omega-7)$ & $64.0 \pm 3.09^{\mathrm{a}}$ & $439 \pm 94.7^{\mathrm{b}}$ \\
$18: 0$ & $338 \pm 9.72^{\mathrm{a}}$ & n.d. \\
18:1 cis $(\omega-9)$ & $640 \pm 8.74^{\mathrm{a}}$ & $1000 \pm 33.1^{\mathrm{b}}$ \\
$18: 1$ trans $(\omega-9)$ & $94.9 \pm 10.7$ & n.d. \\
$18: 2(\omega-6)$ & $62.8 \pm 45.2$ & n.d. \\
$18: 3(\omega-3)$ & n.d. & n.d. \\
$20: 4(\omega-6)$ & n.d. & n.d. \\
$20: 5(\omega-3)$ & $161 \pm 17.0^{\mathrm{a}}$ & $577 \pm 26.8^{\mathrm{b}}$ \\
$22: 5(\omega-3)$ & n.d. & n.d. \\
$22: 6(\omega-3)$ & $1484 \pm 224^{\mathrm{a}}$ & $728 \pm 40.9^{\mathrm{b}}$ \\
Total $\omega-3$ & $1645 \pm 241^{\mathrm{a}}$ & $1304 \pm 67.7^{\mathrm{b}}$ \\
Total $\omega-6$ & $62.8 \pm 45.2^{\mathrm{a}}$ & n.d. \\
Total $\omega-7$ & $64.0 \pm 3.09^{\mathrm{a}}$ & $439 \pm 94.7^{\mathrm{b}}$ \\
Total $\omega-9$ & $735 \pm 19.5^{\mathrm{a}}$ & $1000 \pm 33.1^{\mathrm{b}}$ \\
Total SFA & $1198 \pm 27.43^{\mathrm{a}}$ & $7578 \pm 41.2^{\mathrm{b}}$ \\
Total MUFA & $799 \pm 22.55^{\mathrm{a}}$ & $1439 \pm 128^{\mathrm{b}}$ \\
$\omega-6 / \omega-3$ & 0.04 & 0.00 \\
\hline
\end{tabular}

n.d.: not detectable; SFA: saturated fatty acids; MUFA: monounsaturated fatty acids; $a, b$ indicate significantly different values between the two fish samples (sardine fillet lipid $v s$. cod liver oil; $p<0.05$ ) according to the Mann-Whitney $U$-test.

Two representative chromatograms of TPL and TNL are shown in Figure 2.

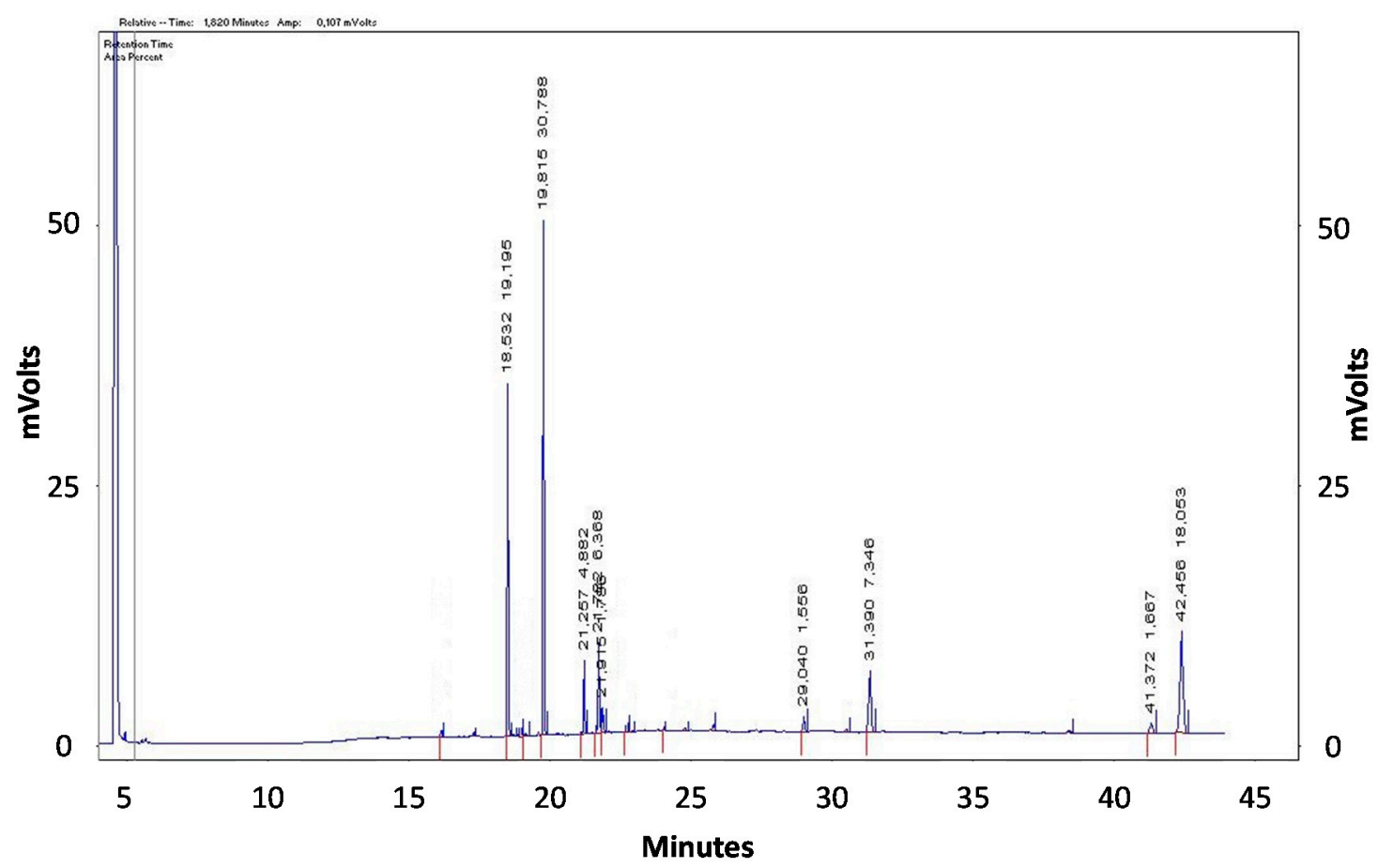

(A)

Figure 2. Cont. 


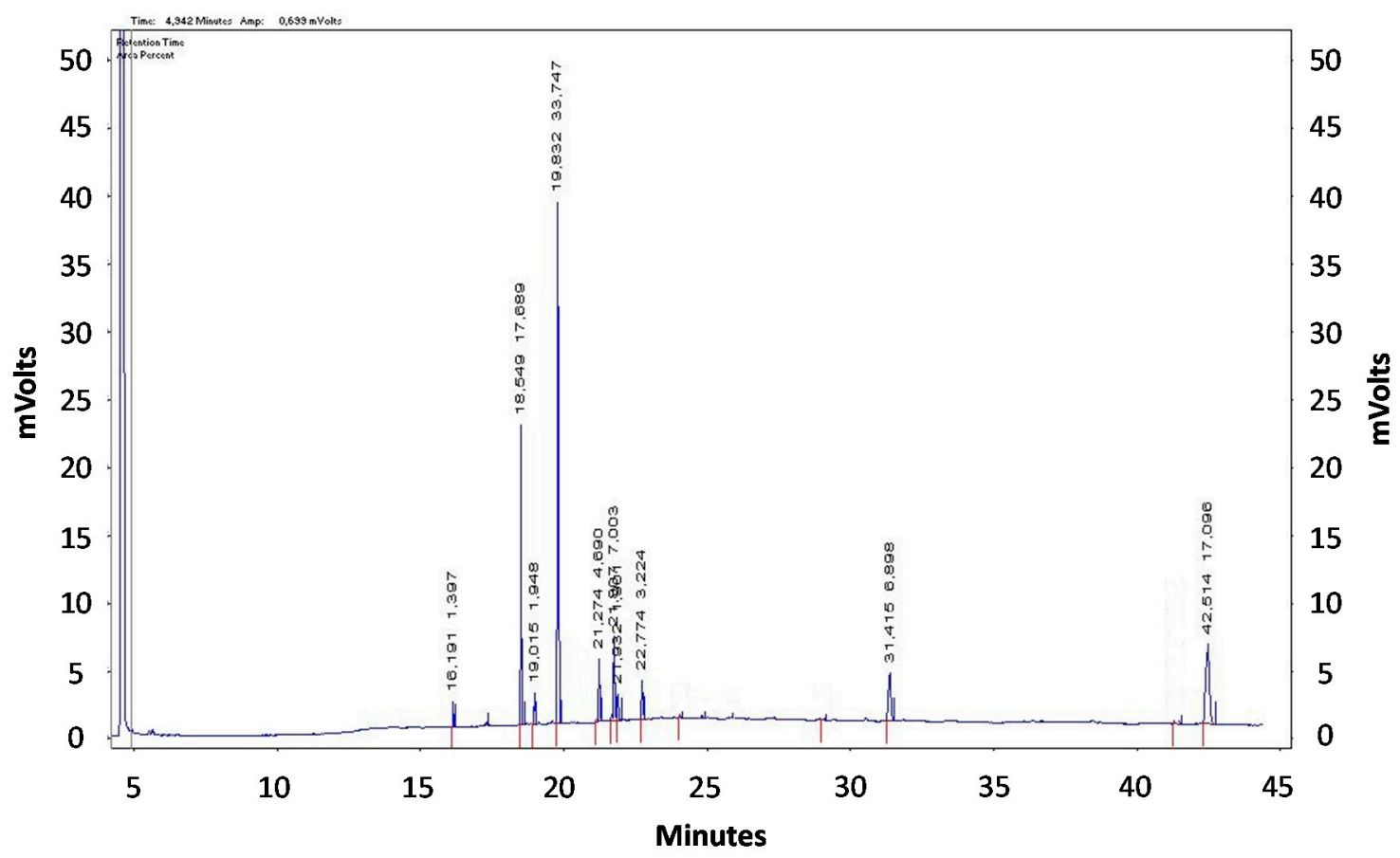

(B)

Figure 2. (A) Representative chromatogram of TPL of sardine fillet and (B) representative chromatogram of TNL of cod liver.

\subsection{Biological Activity of Sardine Fillet and Cod Liver TPL}

The extracted TPL were tested for their ability to induce washed rabbit platelet aggregation or inhibit the PAF-induced washed rabbit platelet aggregation and the equivalent to PAF EC 50 and IC $_{50}$ values of TPL of both samples were expressed as $\mu \mathrm{g}$. TPL of sardine fillet was found to exert aggregatory activity (equivalent to PAF EC $\mathrm{C}_{50}=21.66 \mu \mathrm{g}$ ). However, the TPL of cod liver was found to exert bimodal effect on platelets, inducing platelet aggregation at lower amounts (equivalent to PAF $\mathrm{EC}_{50}=15.59 \mu \mathrm{g}$ ) and inhibiting the PAF-induced platelet aggregation at high ones $\left(\mathrm{IC}_{50}=78.47 \mu \mathrm{g}\right)$. Such bimodal biological activity could be attributed to the fact that TPL is a mixture of lipid molecules that can potentially act either as PAF antagonists (inhibiting PAF activity), or as PAF agonists (inducing platelet aggregation). The overall biological activity observed depends on both the relative ability of each molecule to aggregate platelets or inhibit the PAF-induced platelet aggregation and also dependent on the relative amount of each molecule in the mixture. With this perspective, a lipid fraction that induces platelet aggregation may also contain lipid molecules with inhibitory activity against PAF action or the opposite could occur.

The presence of PAF antagonists (inhibit PAF activity), such as of those present in TPL of cod liver oil, in various foodstuffs is of major importance in terms of nutritional value against CVDs, considering the critical role of platelet activation and thrombosis in the progression of atherogenesis. Moreover, protective intervention studies against atherogenesis have shown that only specific PAF antagonists/inhibitors [27], fish polar lipids [9], olive oil polar lipids [28] and statins [29] are able to reduce atherogenesis in vivo. Therefore the existence in cod liver oil of polar lipid micro-constitutes with antagonist properties against PAF underlines the in vitro antithrombotic potentiality of the 
aforementioned fish oil. Furthermore, sardine fillet TPL exhibited PAF agonistic biological activity. When lipids act as agonists of PAF they actually act as PAF (PAF-like analogs), inducing platelet aggregation. However, these PAF-like analogs are less potent than PAF and could potentially behave as relative PAF antagonists [30].

Additionally, PAF agonists (with aggregatory activity) have been found to have better in vivo antithrombotic activity than PAF-inhibitors [9,31]. With these in mind, sardine fillet TPL, containing PAF agonists, could be considered of increased nutritional value in terms of cardioprotection.

\subsection{TLC Separation of Polar Lipids and Biological Activity of Sardine Fillet and Cod Liver TLC Polar} Lipid Fractions

The TPL of both samples; sardine fillet and cod liver, were further separated by preparative TLC (Figure 3).

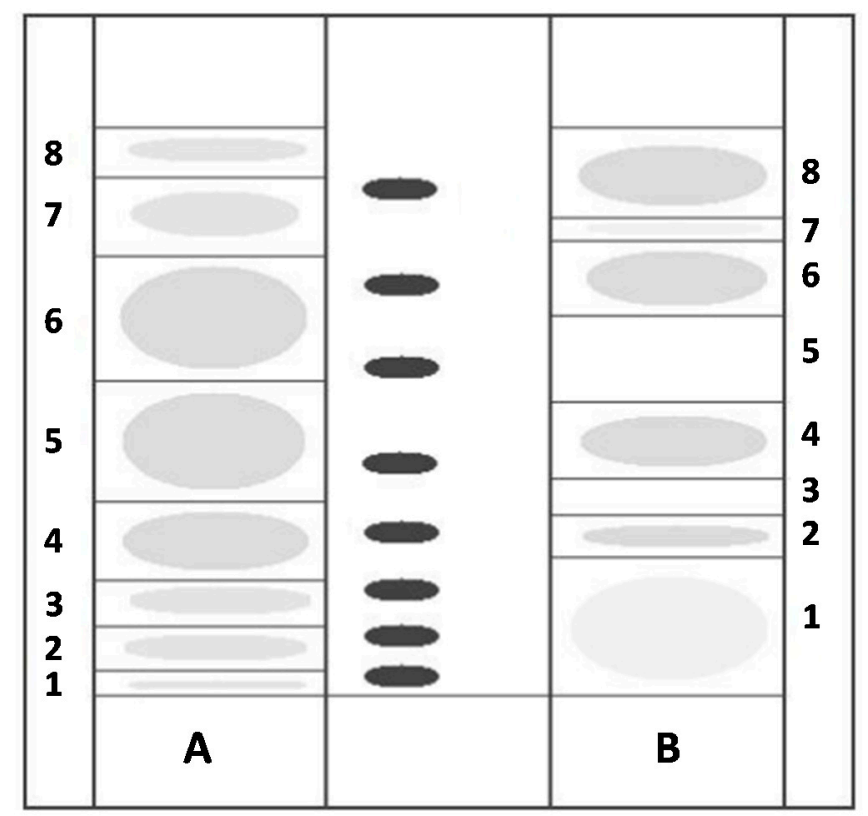

Figure 3. Typical profile of the total polar lipids (TPL) separation on preparative TLC: (A) sardine fillet; (B) cod liver oil (L-PC: lyso-phosphatidylcholine; SM: sphingomyelin; PI: phosphatidylinositol; PC: phosphatidylcholine; PS: phosphatidyloserine; L-PE: lyso-phosphatidylethanolamine; PE: phosphatidylethanolamine; PA: phosphatidic acid; CL: cardiolipin). The elution system used for the separation of total polar lipids was chloroform: methanol: water 65:35:6 $(v / v / v)$.

Eight TLC polar lipid fractions from both samples, sardine fillet and cod liver, were obtained and tested for their ability to induce washed rabbit platelet aggregation or inhibit the PAF-induced platelet aggregation. The equivalent to $\mathrm{PAF}^{\mathrm{E} \mathrm{C}_{50}}$ and $\mathrm{IC}_{50}$ values of each lipid fraction from both samples, sardine fillet and cod liver, were expressed as $\mu \mathrm{g}$ of the lipid fraction and are shown in Figure 4.

TLC polar lipid fractions of sardine fillet and cod liver exhibited the same type of biological activity. More specifically, all TLC polar lipid fractions of both samples exhibited inhibitory properties (PAF antagonists) apart from lipid fraction 1, which showed aggregatory properties (PAF agonists) (Figure 4). 


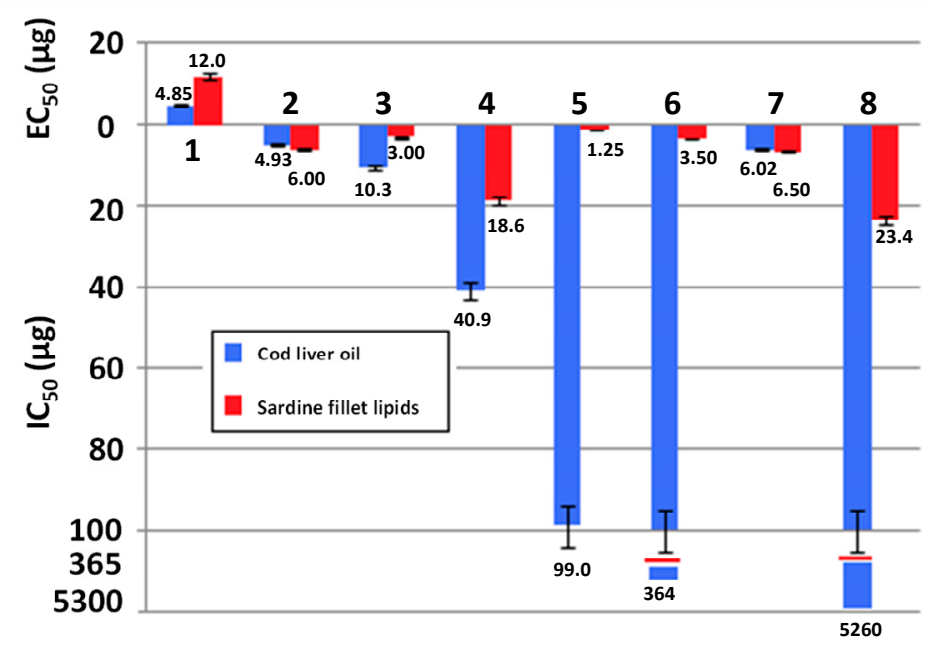

TLC polar lipid fractions

Figure 4. Biological activities (equivalent to PAF equivalent concentration for fifty percent aggregation $\left(\mathrm{EC}_{50}\right)$ and inhibitory concentration for fifty percent inhibition ( $\left.\mathrm{IC}_{50}\right)$ values) of TLC polar lipid fractions of cod liver oil and sardine fillet, expressed as $\mu \mathrm{g}$ of corresponding lipid fraction. All data are the mean \pm SD (95\% confidence levels) of three replicate experiments. $\mathrm{EC}_{50}$ accounts for the amount (expresses as $\mu \mathrm{g}$ ) of each lipid fraction inducing aggregation equivalent to $50 \%$ PAF-induced aggregation $\left(29.59 \times 10^{-11} \mathrm{M}\right.$, final concentration in the cuvette). $\mathrm{IC}_{50}$ accounts for the amount of each lipid fraction (given here as actual mass in $\mu \mathrm{g})$ inhibiting $50 \%$ PAF-induced aggregation $\left(29.59 \times 10^{-11} \mathrm{M}\right.$, final concentration in the cuvette).

TPL of sardine fillet exhibited aggregatory activity, which can be attributed to TLC lipid fraction 1 (Figure 4), while TPL of cod liver showed aggregatory activity at low amounts, which can be attributed to TLC polar lipid fraction 1 and inhibitory activity at higher amounts, which can be attributed to the rest TLC polar lipid fractions 2, 3, 4, 5, 6, 7 and 8 (Figure 4).

The TLC polar lipid fractions of sardine fillet with the most potent inhibitory activity were found to be TLC lipid fractions 5, 3 and 6 with lipid fraction 5 having the lowest $\mathrm{IC}_{50}$ value $(1.25 \mu \mathrm{g})$, thus the most potent inhibitory activity and therefore the most potent PAF antagonists. TLC polar lipid fractions of cod liver with the most potent inhibitory activity were found to be TLC lipid fractions 2 and 7, with fraction 2 having the lowest $\mathrm{IC}_{50}$ value $(4.93 \mu \mathrm{g})$, thus the most potent inhibitory activity and therefore the most potent PAF antagonists.

Comparing the biological activities of the TLC polar lipid fractions of the two fish samples, with similar $R_{\mathrm{f}}$ values, it could be suggested that TLC polar lipid fraction 2 of cod liver, which elutes in the area of phosphatidylcholine (PC) (Figure 3), exhibited statistically significantly lower IC50 values $(p<0.05)$ in comparison to analogues TLC polar lipid fractions of sardine fillet (TLC polar lipid fraction 4) (Figures 2 and 3). Thus, TLC polar lipid fraction 2 of cod liver contains more potent PAF antagonists than the ones of sardine fillet. This result is in good agreement with recent work of our group on polar lipid fractions of sea bass (Dicentrarchus labrax), where several PC species have been structurally identified, suggesting that PC derivatives act as antagonists of PAF-induced platelet activation [13]. 


\section{Experimental Section}

\subsection{Reagents}

All reagents and solvents were of analytical grade purchased from Merck (Darmstadt, Germany). Fatty acid methyl ester standards bought individually were of GC-quality and supplied by Sigma-Aldrich (St. Louis, MO, USA), as well as bovine serum albumin (BSA) and PAF. Chromatographic material used for thin layer chromatography (TLC) was silica gel G-60 supplied by Merck and polar lipid standards used for TLC was a mix standard of hen egg yolk supplied by Sigma-Aldrich. Platelet aggregation was measured in a Chrono-Log aggregometer (model 400-VS) coupled to a Chrono-Log recorder and the gas chromatographer used was a Shimadzu CLASS-VP (GC-17A) (Kyoto, Japan) equipped with a split/splitless injector and flame ionization detector.

\subsection{Sardine (Sardina pilchardus) and Cod Liver Oil Sampling}

One kilogram (1 kg) of raw Greek sardines (Sardina pilchardus) were purchased from a local shop and transported to the laboratory in ice. Individual fish weighed $20 \pm 2.0 \mathrm{~g}$. Raw fish were washed and filleted after fish head, scales, viscera, backbone, skin and tail were removed. Then, $330 \mathrm{~g}$ of raw fish fillets were pooled together and that was the sardine sample.

Cod liver oil was purchased from Seven Seas Ltd, Merck.

\subsection{Isolation of Fish Total Lipids of Sardine (Sardina pilchardus) and Cod Liver Oil}

Total lipids (TL) of sardine fillets were extracted according to the Bligh-Dyer method [21]. For each extraction, a sample of $110 \mathrm{~g}$ of fish fillet was obtained by combining several fish fillets and this sampling procedure was carried out in triplicate. One tenth of the TL samples were stored in sealed vials at $-20{ }^{\circ} \mathrm{C}$. The rest TL was further separated into total polar lipids (TPL) and total neutral lipids (TNL) using the counter-current distribution method [22]. TNL and one tenth of TPL were stored in sealed vials at $-20{ }^{\circ} \mathrm{C}$ for further analysis. The rest of TPL were further separated by preparative TLC and the obtained TLC polar lipid fractions (containing glycolipids and phospholipids) were stored in sealed vials at $-20{ }^{\circ} \mathrm{C}$ for further analysis.

A quantity of $30 \mathrm{~mL}$ of cod liver oil was separated into TPL and TNL by counter-current distribution method [22]. TNL and one tenth of TPL were stored in sealed vials at $-20{ }^{\circ} \mathrm{C}$ for further analysis. The rest of TPL were further separated by preparative TLC and the obtained TLC polar lipid fractions were stored in sealed vials at $-20{ }^{\circ} \mathrm{C}$ for further analysis.

\subsection{Gas Chromatography Analysis}

Fatty acid methyl esters of TPL and TNL of sardine fillets and cod liver were prepared using a solution $0.5 \mathrm{~N} \mathrm{KOH}$ in $\mathrm{CH}_{3} \mathrm{OH} 90 \%$ and extracted with $n$-hexane. The fatty acid analysis was carried out using the internal standard method, as described extensively by Nasopoulou et al., 2011 [32]. The gas chromatographer used was a Shimadzu CLASS-VP (GC-17A) (Kyoto, Japan) equipped with a split/splitless injector and flame ionization detector. 
Separation of fatty acid methyl esters was achieved on an Agilent J\&W DB-23 fused silica capillary column $\left(60 \mathrm{~m} \times 0.251 \mathrm{~mm}\right.$ i.d., $0.25 \mu \mathrm{m}$; Agilent). The oven temperature program was: $120{ }^{\circ} \mathrm{C}$ for $5 \mathrm{~min}$, raised to $180{ }^{\circ} \mathrm{C}$ at $10{ }^{\circ} \mathrm{C} \cdot \mathrm{min}^{-1}$, then to $220{ }^{\circ} \mathrm{C}$ at $20^{\circ} \mathrm{C} \cdot \mathrm{min}^{-1}$ and finally isothermal at $220{ }^{\circ} \mathrm{C}$ for $30 \mathrm{~min}$. The injector and detector temperatures were maintained at 220 and $225{ }^{\circ} \mathrm{C}$, respectively. The carrier gas was high purity helium with a linear flow rate of $1 \mathrm{~mL} \cdot \mathrm{min}^{-1}$ and split ratio $1: 50$. Fatty acid methyl esters were identified using fatty acid methyl esters standards by matching retention times of the relative peaks.

\subsection{Fractionation of TPL by TLC}

The TLC glass plates $(20 \times 20 \mathrm{~cm})$ were coated with silica gel G-60 and activated by heating at $120{ }^{\circ} \mathrm{C}$ for $60 \mathrm{~min}$. The thickness of the TLC plates was $1.0 \mathrm{~mm}$ (preparative TLC). Approximately $50 \mathrm{mg}$ of TPL of sardine fillet (Sardina pilchardus) and $25 \mathrm{mg}$ of TPL of cod liver were applied to the TLC plates. A developing system consisting of chloroform:methanol:water 65:35:6 ( $v / v / v)$ was utilized for the separation of TPL. The plates were stained under iodine vapors. Eight bands, either for sardine fillet or for cod liver, appeared after the separation of TPL by TLC. After the vaporization of iodine vapors, the bands were scraped and lipids were extracted from the silica gel according to the Bligh-Dyer method [21]. The chloroform phase was evaporated to dryness under nitrogen and lipids were weighed, redissolved in $1 \mathrm{~mL}$ chloroform:methanol $1: 1(v / v)$ and stored at $-20{ }^{\circ} \mathrm{C}$, as described earlier [8].

\subsection{Biological Assay of the in Vitro Antithrombotic Properties}

The TLC polar lipid fractions of sardine fillet and cod liver were tested for their biological activity according to the washed rabbit platelet aggregation assay [11]. Briefly, the samples being examined and PAF were dissolved in $2.5 \mathrm{mg}$ of bovine serum albumin (BSA) per $\mathrm{mL}$ of saline. Various amounts of the sample being examined, ranging from 1.25 to $7395 \mu \mathrm{g}$, were added into the aggregometer cuvette and their ability to aggregate washed rabbit platelets or to inhibit PAF-induced aggregation was determined. In order to determine the aggregatory efficiency of either PAF or the samples being examined, the maximum reversible aggregation was evaluated and the $100 \%$ aggregation was determined. The plot of the percentage of the maximum reversible aggregation (ranging from $20 \%$ to $80 \%$ ) versus different concentrations of the aggregatory agent was linear. From this curve, the concentration of the aggregatory agent, which induces $50 \%$ of the maximum reversible PAF-induced aggregation, is calculated. This value is defined as the amount of the sample that induces an equivalent to PAF EC50, namely equivalent concentration for $50 \%$ aggregation.

In order to determine the inhibitory properties of the samples, various amounts of the sample being examined, ranging from 1.25 to $7395 \mu \mathrm{g}$, were added into the aggregometer cuvette and their ability to inhibit PAF-induced aggregation was determined. The platelet aggregation induced by PAF $\left(2.95 \times 10^{-11} \mathrm{M}\right.$ final concentration in the cuvette) was measured as PAF-induced aggregation, in washed rabbit platelets before (considered as $0 \%$ inhibition) and after the addition of various amounts of the sample being examined. Consequently, the plot of $\%$ inhibition (ranging from $20 \%$ to $80 \%$ ) versus different concentrations of the sample is linear. From this curve, the concentration of the sample, which 
inhibited 50\% PAF-induced aggregation, is calculated. This value is defined as $\mathrm{IC}_{50}$, namely inhibitory concentration for $50 \%$ inhibition.

\subsection{Desensitization Experiment}

Desensitization experiment was carried out according to the method of Lazanas et al. (1988) [33]. Briefly, platelets were desensitized by the addition of PAF or the examined aggregatory agent to the platelet suspension at a concentration that caused same size reversible aggregation. Next, stimulation was induced immediately after complete disaggregation by the addition of the same concentration of PAF or the examined aggregatory agent. It was observed that the second addition of the aggregatory agent caused less aggregation than the initial, due to the desensitization of platelets when they act through the same receptor. In cross-desensitization experiments the same experiment was repeated twice with the addition of different aggregatory agent each time [34].

\subsection{Statistical Analysis}

Chemical analyses were carried out six times and all results were expressed as mean \pm SD in all cases. The Wilcoxon sign test was performed to evaluate significant differences within the same group, while the Mann-Whitney $U$-test was performed to evaluate significant differences among different groups. Differences were considered to be statistically significant when $p$-value was less than 0.05 . Data were analyzed using a statistical software package (SPSS for Windows, 20.0, 2012, SPSS Inc., Chicago, IL, USA).

\section{Conclusions}

In the research reported here, we have focused on the polar lipids of cod liver and sardine fillet, since previous studies have proved that the antithrombotic properties of foodstuffs are mainly attributed to polar lipid microconstituents. These two fish species have been chosen since sardine is the raw ingredient in the manufacture of dietary supplements of $\omega-3$ PUFA and cod liver oil is widely used as a health supplement. The significant differences observed between the high content of TPL of sardine fillet lipids and the low TPL content of cod liver oil could be attributed to the different extraction methods that are used to deliver lipids from these two fish species. The use of solvents of high polarity in the lipid extraction of sardine tissue has increased the levels of the extracted polar lipids in comparison to the less selective way used for lipid extraction of cod and production of the nutraceutical cod liver oil. It was also shown here that neutral lipids are the dominant lipid class in cod liver oil, probably due to the wet rendering process that is used in the manufacture of cod liver oil. This process does not include the use of polar solvents and it thus leads to an extract with much lower levels of antithrombotic polar lipids [35].

The amounts of most fatty acids in TPL of cod liver were found to be significantly lower in comparison to the amounts of fatty acids in TPL of sardine fillet. TPL of sardine fillet was also found to exert aggregatory properties, while TPL of cod liver was found to exert bimodal effect on platelets, inducing platelet aggregation at lower amounts and inhibiting the PAF-induced platelet aggregation at high ones. Conclusively, in this work, it was found that cod liver oil contains few polar lipids and these 
polar lipids are less in vitro antithrombotic than the corresponding ones obtained from sardine. In addition, the re-evaluation of the extraction method used in the production of cod liver oil needs to be carried out in order to increase the fraction of polar lipids obtained. This study is the first in vitro study confirming that cod liver oil contains less polar lipids and has lower activities against atherogenesis than sardine polar lipids.

Our in vitro results should correspond to analogous favorable in vivo results as previous studies of our group has shown, i.e., we have found that polar lipids of sea bream have strong antithrombotic properties both in vitro [8] and in vivo [9]. Such in vivo studies should be the best way forward to confirm that the examined lipid fractions in this paper have such antithrombotic actions that could be transferable to physiological or pathological effects. The first results, though, as presented here are encouraging and our future work will focus on in vivo studies of the lipids of sardine and cod.

\section{Author Contributions}

Gregory Morphis, Aggeliki Kyriazopoulou, Eleni Sioriki and Constantina Nasopoulou performed the experimental part of the work, whereas Constantinos A. Demopoulos and Ioannis Zabetakis contributed the original idea of this project and edited the manuscript.

\section{Conflicts of Interest}

The authors declare no conflict of interest.

\section{References}

1. Kris-Etherton, P.M.; Harris, W.S.; Appel, L.J. Fish consumption, fish oil, $\omega-3$ fatty acids and cardiovascular disease. Arterioscler. Thromb. Vasc. Boil. 2003, 23, 20-31.

2. Din, J.N.; Newby, D.E.; Flapan, A.D. $\omega-3$ Fatty acids and cardiovascular disease-fishing for a natural treatment. Br. Med. J. 2004, 328, 30-35.

3. Kristensen, S.D.; Iversen, A.M.; Schmidt, E.B. n-3 Polyunsaturated fatty acids and coronary thrombosis. Lipids 2001, 36, S79-S82.

4. Mori, T.A.; Beilin, L.J.; Burke, V.; Morris, J.; Ritchie, J. Interactions between dietary fat, fish, and fish oil and their effects on platelet function in men at risk of cardiovascular disease. Arterioscler. Thromb. Vasc. Biol. 1997, 17, 279-286.

5. Rementzis, J.; Antonopoulou, S.; Demopoulos, C.A. Identification and study of gangliosides from Scomber scombrus muscles. J. Agric. Food Chem. 1997, 45, 611-615.

6. Panayiotou, A.; Samartzis, D.; Nomikos, T.; Fragopoulou, E.; Karantonis, H.C.; Demopoulos, C.A.; Demopoulos, C.A.; Zabetakis, I. Lipid fractions with aggregatory and antiaggregatory activity toward platelets in fresh and fried cod (Gadus morhua): Correlation with platelet-activating factor and atherogenesis. J. Agric. Food Chem. 2000, 48, 6372-6379.

7. Nomikos, T.; Karantonis, H.C.; Skarvelis, C.; Demopoulos, C.A.; Zabetakis, I. Antiatherogenic properties of lipid fractions of raw and fried fish. Food Chem. 2006, 96, 29-35. 
8. Nasopoulou, C.; Nomikos, T.; Demopoulos, C.A.; Zabetakis, I. Comparison of antiatherogenic properties of lipids obtained from wild and cultured sea bass (Dicentrarchus labrax) and gilthead sea bream (Sparus aurata). Food Chem. 2007, 100, 560-567.

9. Nasopoulou, C.; Karantonis, H.C.; Perrea, D.N.; Theocharis, S.E.; Iliopoulos, D.G.; Demopoulos, C.A.; Zabetakis, I. In vivo anti-atherogenic properties of cultured gilthead sea bream (Sparus aurata) polar lipid extracts in hypercholesterolaemic rabbits. Food Chem. 2010, 120, 831-836.

10. Roger, V.L.; Go, A.S.; Lloyd-Jones, D.M.; Benjamin, E.J.; Berry, J.D.; Borden, W.B.; Bravata, D.M.; Dai, S.; Ford, E.S.; Fox, C.S.; et al. Executive Summary: Heart disease and stroke statistics - 2012 update. Circulation 2012, 125, 188-197.

11. Demopoulos, C.A.; Pinckard, R.N.; Hanahan, D.J. Platelet-activating factor. Evidence for 1-O-alkyl-2-acetyl-sn-glyceryl-3-phosphoryl-choline as the active component (a new class of lipid chemical mediators). J. Biol. Chem. 1979, 254, 9355-9358.

12. Demopoulos, C.A.; Karantonis, H.C.; Antonopoulou, S. Platelet activating factor-A molecular link between atherosclerosis theories. Eur. J. Lipid Sci. Technol. 2003, 105, 705-716.

13. Nasopoulou, C.; Smith, T.; Detopoulou, M.; Tsikrika, C.; Papaharisis, L.; Barkas, D.; Zabetakis, I. Structural elucidation of olive pomace fed sea bass (Dicentrarchus labrax) polar lipids with cardioprotective activities. Food Chem. 2014, 145, 1097-1105.

14. Nasopoulou, C.; Gogaki, V.; Stamatakis, G.; Papaharisis, L.; Demopoulos, C.A.; Zabetakis, I. Evaluation of the in vitro anti-atherogenic properties of lipid fractions of olive pomace, olive pomace enriched fish feed and gilthead sea bream (Sparus aurata) fed with olive pomace enriched fish feed. Mar. Drugs 2013, 11, 3676-3688.

15. De Leonardis, A.; Macciola, V. A study on the lipid fraction of Adriatic sardine filets (Sardina pilchardus). Food/Nahrung 2004, 48, 209-212.

16. Duan, Z.; Kangsen, M.; Qinghui, A.; Milley, E.J.; Lall, P.S. Lipid and fatty acid compositions of cod (Gadus morhua), haddock (Melanogrammus aeglefinus) and halibut (Hippoglossus hippoglossus). J. Ocean Univ. China 2010, 9, 381-388.

17. Kurlansky, M. Cod: A Biography of the Fish That Changed the World; Penguin Group: London, UK, 1999.

18. Lie, O.E.; Lied, E.; Lambertsen, G. Liver retention of fat and of fatty acids in cod (Gadus morhua) fed different oils. Aquaculture 1986, 59, 187-196.

19. Calvano, C.D.; Zambonin, C.G.; Foti, C.; Cassano, N.; Vena, G.A. A matrix assisted laser desorption ionization time-of-flight mass spectrometry investigation to assess the composition of cod liver oil based products which displayed a different in vivo allergenic power. Food Chem. Toxicol. 2008, 46, 3580-3585.

20. Rice, R. Fish Oils/Dietary Importance. In Encyclopedia of Food Sciences and Nutrition; The Fish Foundation: Tiverton, UK, 2003.

21. Bligh, E.G.; Dyer, W.J. A rapid method of total lipid extraction and purification. Can. J. Biochem. Physiol. 1959, 37, 911-917.

22. Galanos, D.S.; Kapoulas, V.M. Isolation of polar lipids from triglyceride mixtures. J. Lipid Res. 1962, 3, 134-137.

23. Swapna, H.C.; Rai, A.K.; Bhaskar, N.; Sachindra, N.M. Lipid classes and fatty acid profile of selected Indian fresh water fishes. J. Food Sci. Technol. 2010, 47, 394-400. 
24. Kim, J.G.; Han, B.W.; Kim, H.S.; Park, C.H.; Chung, I.K.; Choi, Y.J.; Kim, J.S.; Heu, M.S. Lipid characteristics of fish frame as a functional lipid resource. J. Korean Soc. Food Sci. Nutr. 2005, 34, 380-388.

25. Ackman, R.G. Nutritional composition of fats in seafoods. Prog. Food Nutr. Sci. 1989, 13, 161-241.

26. Bandarra, N.M.; Batista, I.; Nunes, M.L.; Empis, J.M.; Christie, W.W. Seasonal changes in lipid composition of sardine (Sardina pilchardus). J. Food Sci. 1997, 62, 40-42.

27. Feliste, R.; Perret, B.; Braqueta, P.; Chap, H. Protective effect of BN 52021, a specific antagonist of platelet-activating factor (PAF acether) against diet-induced cholesteryl ester deposition in rabbit arta. Atherosclerosis 1989, 78, 151-158.

28. Karantonis, H.C.; Antonopoulou, S.; Perrea, D.N.; Sokolis, D.P.; Theocharis, S.E.; Kavantzas, N.; Iliopoulos, D.G.; Demopoulos, C.A. In vivo antiatherogenic properties of olive oil and its constituent lipid classes in hyperlipidemic rabbits. Nutr. Metab. Cardiovasc. Dis. 2006, 16, 174-185.

29. Wierzbicki, A.S.; Poston, R.; Ferro, A. The lipid and non-lipid effects of statins. Pharmacol. Ther. 2003, 99, 95-112.

30. Stafforini, D.M.; Zimmerman, G.A. Unraveling the PAF-AH/Lp-PLA 2 controversy. J. Lipid Res. 2014, 55, 1811-1814.

31. Tsantila, N.; Karantonis, H.C.; Perrea, D.N.; Theocharis, S.E.; Iliopoulos, D.G.; Antonopoulou, S.; Demopoulos, C.A. Antithrombotic and antiatherosclerotic properties of olive oil and pomace polar extracts in rabbits. Med. Inflamm. 2007, 2007, 1-11.

32. Nasopoulou, C.; Stamatakis, G.; Demopoulos, C.A.; Zabetakis, I. Effects of olive pomace and olive pomace oil on growth performance, fatty acid composition and cardioprotective properties of gilthead sea bream (Sparus aurata) and sea bass (Dicentrarchus labrax). Food Chem. 2011, 129, $1108-1113$.

33. Lazana, L.K.; Lazanas, M.; Koussissis, S.; Tournis, S.; Demopoulos, C.A. PAF of Biological fluids in desease: Blood levels in allergic rhinitis. Haematologica 1988, 73, 379-382.

34. Antonopoulou, S.; Demopoulos, C.A.; Andrikopoulos, N.K. Lipid separation from Urtica dioica: Existence of platelet-activating factor. J. Agric. Food Chem. 1996, 44, 3052-3056.

35. Bimbo, A.P. Sources of marine oils. In Marine Oils, from Sea to Pharmaceuticals, 1st ed.; Nova Science Publishers: Hauppauge, NY, USA, 2015; pp. 1.

(C) 2015 by the authors; licensee MDPI, Basel, Switzerland. This article is an open access article distributed under the terms and conditions of the Creative Commons Attribution license (http://creativecommons.org/licenses/by/4.0/). 